\title{
Un regard sur la domesticité juvénile en Haïti
}

\author{
IRDÈLE LUBIN
}

\section{Résumé}

Enfants en domesticité, enfants en service, ou restavèk (reste avec), telles sont les appellations reçues par des petites filles et des petits garçons qui sont placés dans des familles d'accueil afin de servir celles-ci. En retour, ces familles d'accueil, doivent leur fournir de quoi satisfaire quelques-uns de leurs besoins primaires (nourriture, logement, vêtement) et éventuellement le paiement des cours dans une école. Maltraités, humiliés, considérés au plus bas de l'échelle familiale, ces enfants seront traités comme de petits esclaves. Ils travaillent à longueur de journée, mais ils sont oubliés et ne font pas l'objet de beaucoup d'intérêt. La législation permettant de leur fournir une certaine protection dans les familles d'accueil n'est pas respectée, leurs droits ne sont pas respectés. Cet article met en évidence la situation des enfants en domesticité en Haïti, la législation existant sur la question, et la façon dont ces enfants vivent leurs droits dans le pays.

\section{Abstract:}

'Domestic Children', 'Service Children' or 'restavèk' ('livein') - such are some of the appellations given to young girls and boys placed in host families in order to serve them. In exchange, these host families are supposed to cater for some of their basic needs (food, shelter, clothing), and in due course, to pay for their school fees. These children are ill treated, humiliated and considered as the lowest of the low in the family. They are treated as young slaves. They toil endlessly from morning to night, and are yet totally forgotten and attract hardly any interest. The legislation providing some degree of protection for them in the host families is totally disregarded and their rights ignored. This article focuses on the plight of Domestic Children in Haiti, the laws applicable to the situ- ation, and the manner in which these children are living an existence deprived of rights in the country.

\section{Introduction}

Un peu partout dans le monde, le travail des enfants a entraîné des débats. Plusieurs dimensions de la question ont été abordées. Elle a suscité des prises de position diverses et variées sur le développement de ces enfants qui, dans bien des cas, mènent des activités trop exigeantes pour leur force physique. La législation sur le travail des enfants porte fondamentalement sur l'âge minimal pour être admis à travailler, la nature des travaux, le nombre d'heures de travail et le salaire. Certains pays légifèrent aussi sur le travail non rémunéré des enfants effectuant des travaux domestiques et ménagers. Mais les conditions dans lesquelles vivent ces enfants ne font pas l'objet de recherche et le fossé entre la loi et la pratique reste grand.

En Haïti, les enfants qui exécutent des tâches domestiques, les enfants en domesticité, poussent des cris de désespoir; mais ils sont oubliés et abandonnés chez des gens qui les maltraitent, qui n'ont pas peur de la loi à cause d'une certaine complicité avec les structures d'application ou du mépris général à l'égard de ces enfants. Qui sont ces enfants? D'où viennent-ils? En regard de la convention sur les droits des enfants, comment viventils leurs droits? C'est à ces questions qu'essaie de répondre cet article qui présente :

- de manière succincte, la situation des enfants en domesticité en Haïti,

- la législation haïtienne sur les enfants en domesticité,

- la façon dont ces enfants vivent leurs droits dans le pays. 


\section{Les enfants en domesticité en Haïti}

Enfants en domesticité ou enfants en service comme les nomme la législation haïtienne, ils ne sont pas très connus dans le monde. Dans certains milieux, ils sont souvent confondus « simplement » avec le groupe des mineurs travailleurs ${ }^{1}$. C'est un problème existant dans la plupart des pays pauvres et même dans certains pays développés ${ }^{2}$; mais il n'a pas fait l'objet de plusieurs recherches. Les enfants domestiques présentent des nuances très importantes selon le contexte et la culture de leur lieu de travail. Ces nuances sont très importantes pour permettre de mieux situer le problème, structurer éventuellement les interventions visant à améliorer les conditions d'existence des enfants concernés ou pour l'éliminer parce que c'est une pratique contraire à la Convention relative aux droits de l'enfant.

En Haïti, certains ${ }^{3}$ ont considéré l'absence de rémunération et les tâches accomplies par les restavèk pour tenter de les définir. Cela ne permet pas de mieux situer le problème; on peut facilement les confondre avec d'autres catégories d'enfants tels les adoptés, les enfants placés provisoirement ou les « pensionnaires » ou même ceux qui vivent chez leurs parents biologiques et qui aident aux travaux ménagers. C'est un fait que " le restavèk fournit un labeur non rémunéré en argent mais plutôt en échange du logement, de la nourriture, du vêtement et de l'éducation $»^{4}$, mais il y a aussi d'autres éléments à considérer pour comprendre le problème de la domesticité.

On peut dire que l'enfant en domesticité est un individu dont l'âge peut être compris entre 6 à 14 ans. La domesticité n'est presque jamais son choix, c'est une décision qui a été prise par ses parents ou un proche dont les faibles moyens économiques ne permettent pas de répondre aux besoins de l'enfant. La domesticité est un moyen de lui procurer un mieux-être par son placement chez une personne vivant dans de meilleures conditions économiques. Généralement, l'enfant est avisé de cette décision juste avant de se rendre chez la personne qui le recevra comme domestique. Il vit sous le toit de cette personne et dessert tous les gens de la demeure moyennant un peu de nourriture et des vêtements; éventuellement on lui paie des cours dans une petite école. Dans la majorité des cas, il s'agit d'une petite fille, les garçons étant moins nombreux à être astreints aux travaux strictement domestiques. L'enfant en domesticité est considéré au plus bas échelon dans cette demeure; ses droits sont très limités pour ne pas dire inexistants et ses devoirs sont énormes. Les deux principales attitudes exigées de lui sont la soumission et la serviabilité. Il est souvent victime d'actes de violence (verbale, physique, psychologique...) de la part de son supposé protecteur ou protectrice.

Il faut éviter de prendre pour des enfants en domesticité tous ceux qui s'occupent des tâches domestiques dans une maison autre que la leur. En ce sens, mentionnons les cas de plusieurs enfants (filles et garçons) qui ne vivent pas chez leurs parents biologiques pour plusieurs raisons telles que l'adoption, le départ prolongé des parents biologiques, l'accessibilité à une école de niveau académique plus ou moins intéressante, l'apprentissage d'un métier etc. Ces cas sont très fréquents en Haïti. Les enfants de ces catégories ne sont pas considérés comme des domestiques, quoique certaines fois ils puissent aider aux travaux domestiques. Dans les cas où ils se sentent considérés comme des restavè , ils ont toujours la possibilité d'aller ailleurs. Ce n'est pas le cas pour les enfants en domesticité. Par ailleurs, bon nombre de ces enfants (dans les deux derniers cas surtout) regagnent leur famille biologique à certaines périodes de l'année. L'enfant en domesticité n'a pas beaucoup de contact avec ses parents biologiques. Il y en a qui ont perdu tout contact avec leurs parents parce que la famille de placement ne les envoie pas toujours visiter leurs parents biologiques. Ils sont ainsi réfugiés dans leur propre pays où même les rencontres avec leurs familles biologiques ou un proche parent s'avèrent difficiles. Leurs parents sont souvent trop pauvres pour consentir des frais de transport.

De manière succincte, pour mieux situer le restavèk haïtien il faut considérer les éléments suivants :

- c'est un enfant généralement placé ou trouvé,

- le but de son placement est de rendre service afin de recevoir en retour des éléments indispensables à sa survie et éventuellement les cours dans une école,

- la période de placement n'est pas définie,

- ses rapports avec les membres de la famille de placement sont fondés sur la domination et l'autorité,

- l'état psychologique et émotionnel est un élément important à considérer aussi.

\section{Législation relative aux enfants en domesticité en Haïti}

La législation haïtienne fait une différence entre enfants en domesticité, mineurs travailleurs et gens de maison.

Appelés enfants en service par la législation haïtienne, dans la réalité de tous les jours, ils sont appelés enfants en domesticité, timoun ki ret kay moun ${ }^{5}$ ou restavè $k^{6}$. D'après le décret mettant à jour le Code du travail du 12 septembre $1961^{7}$, l'enfant en service est celui âgé de 12 à 15 ans (articles 341 et 350) «confié à une famille pour être employé à des travaux domestiques ». D'après l'article 350 de ce même décret, « dès l'âge de 15 ans, l'enfant en service sera considéré comme domestique à gages et recevra un salaire équivalent à celui payé aux domestiques à gages travaillant dans les mêmes conditions ». 
L'enfant en service ou en domesticité tel que défini ne reçoit pas, comme cela est mentionné antérieurement, un salaire pour les services rendus à la famille de placement. L'article 345 du décret précédemment cité, mentionne les besoins et les obligations auxquels ces enfants ont droit dans leur famille de placement. " Toute personne qui a un ou plusieurs enfants à son service contracte envers eux l'obligation de les traiter en bon père de famille, de leur fournir un logement décent, des vêtements convenables, une nourriture saine et suffisante de les inscrire obligatoirement à un centre d'enseignement scolaire ou professionnel en leur permettant de suivre régulièrement les cours dispensés par ce centre et de leur procurer de saines distractions ». Il est évident que les enfants en domesticité n'ont pas pu, pour différentes raisons, avoir ces besoins indispensables à leur développement chez leurs parents biologiques.

Le mineur travailleur, par contre, est âgé de 15 à 18 ans (article 337) et travaille dans un établissement agricole, industriel ou commercial. Il " bénéficie ${ }^{8}$ » des mêmes droits et des mêmes obligations que les majeurs en vertu de la législation du travail.

Les gens de maison (article 254), appelés de manière générale bonnes ou garçons de cour, sont « ceux qui se consacrent de façon habituelle et continue aux travaux de nettoyage, de jardinage, d'entretien ou à tous autres travaux domestiques propres à un foyer ou à tout autre lieu de résidence ou d'habitation particulière ou dans une institution privée ou publique de bienfaisance et qui ne comportent ni bénéfice, ni opération commerciale pour l'employeur ou les membres de sa famille ».

Si la législation haïtienne fait une différence entre ces trois catégories de personnes, la réalité n'en fait pas vraiment. L'enfant en domesticité peut être considéré à la fois comme un mineur travailleur eu égard à son âge et comme bonne à tout faire ou gens de maison vu la nature des tâches auxquelles il est occupé habituellement. Par ailleurs, cela fait plus de 40 ans depuis que cette législation sur les enfants en service existe, mais elle n'a pas contribué à une amélioration du sort de ces enfants. Comme le souligne certains auteurs ${ }^{9}$, le fossé entre la pratique et la loi reste très large.

\section{Âge, sexe et provenance des enfants en domesticité}

Les enfants en domesticité en Haiti, les restavèk, sont majoritairement de sexe féminin. D'après une enquête de l'Institut psycho-social de la famille ${ }^{10}$, « l'âge moyen des enfants en domesticité juvénile se situe entre 11 et 14 ans ». Il est courant de rencontrer des petites filles de 6 à 10 ans qui commencent très tôt à vivre dans la domesticité. Dans l'enquête de l'IPSOFA, les domestiques rencontrés sont majoritairement (à $75 \%$ ) des petites filles.
Les enfants placés en domesticité viennent pour la grande majorité des familles pauvres des zones rurales réfugiées certaines fois dans des zones péri-urbaines à la recherche d'un mieux-être. Ils forment, dans la grande majorité, les bidonvilles et les quartiers défavorisés du pays. Dans son livre sur la famille haitienne ${ }^{11}$, L. Bijoux mentionne que « les enfants de service, dont les parents sont généralement des paysans pauvres, sont confiés aux familles plus ou moins aisées des villages et des villes ». Ces familles espèrent que leurs enfants seront bien traités. Elles comptent ainsi, comme le souligne L. Bijoux, sur le sens de solidarité et d'équité des familles d'accueil qui promettent toujours une meilleure éducation en compensation des travaux domestiques rendus par les enfants. Pour Despeignes, « les trois quarts du lumpen-prolétariat proviennent de l'immense armée des domestiques ${ }^{12}$ ». L'IPSOFA a recensé $81 \%$ de ces parents qui viennent des zones rurales. D'après Serge Henri-Vieux ${ }^{13}$, la domesticité touche $80 \%$ des enfants des couches défavorisées. Ces enfants sont placés par leurs parents ou un proche en l'absence de ceux-ci. D'après l'enquête de l'IPSOFA, $82 \%$ des enfants se trouvent dans cette situation.

\section{Un état de la question}

De manière générale, il est constaté une dégradation de la situation économique en Haïti où la grande majorité des familles vit dans un état de misère. Les enfants, dans cette situation, sont les plus concernés et les enfants en service le sont encore davantage. La grande majorité des enfants placés en domesticité viennent de ces familles, comme d'ailleurs il est remarqué dans les cas des mineurs travailleurs ${ }^{14}$. Combien y a-t-il d'enfants en service en Haïti? Il est difficile d'avancer un chiffre. Il n'y a pas eu de recensement dans le pays depuis vingt ans. Certains organismes font de petites enquêtes mais, connaissant le pouvoir et la finalité de l'utilisation des chiffres dans les pays du tiers monde, il est recommandé de les utiliser avec précaution.

Le dernier recensement date de 1982 et, en 1984, l'Institut haïtien de statistiques (IHSI) ${ }^{15}$ a estimé la population des enfants en domesticité à 109000 dont 65000 filles et 44000 garçons. D'autres sources, comme le $\mathrm{BIT}^{16}$, avancent le chiffre de 182800 enfants de 10 à 14 ans officiellement reconnus comme enfants travailleurs. Est-ce que les enfants en domesticité font partie de ce chiffre? On ne saurait le dire. Mais il faut se rappeler que, généralement, ils sont considérés dans cette catégorie. Lors d'un Forum sur l'enfance et la violence en Haïti, tenu les 18 et 19 octobre $1995^{17}$, le psychiâtre Legrand Bijoux a parlé dans une présentation sur les enfants en 
domesticité de 200000 à 300000 enfants vivant dans cette situation. D'après l'UNICEF, cité par Serge Henri-Vieux au cours du même forum, il existe 130000 enfants vivant en domesticité. Depuis la dernière estimation de l'IHSI en 1984, il n'y a pas de chiffre officiel sur le nombre d'enfants vivant en domesticité. Mais la tendance est de les confondre avec des enfants des zones marginales. Notons que ceux-ci peuvent vivre dans les mêmes conditions socio-économiques que les enfants en domesticité. Mais ils ne sont pas placés en service. Cette nuance est importante, car les enfants de la rue par exemple disent préférer la vie de la rue à la domesticité. Ils n'acceptent pas d'être considérés comme des enfants en service. En ce sens, il existe des enfants qui vivent chez eux, mais leurs conditions économiques sont pareilles à celles des enfants en domesticité ou pires. Mais le «bonheur » est qu'ils ne sont pas placés. La domesticité représente la situation ou la condition la plus dégradante de l'existence humaine dans la réalité haïtienne.

\section{Conditions de vie des enfants en domesticité}

Dans la grande majorité des cas, les conditions d'existence des enfants en domesticité ne sont comparables à aucune autre. Ils travaillent à longueur de journée, de 10 à 15 heures par jour ${ }^{18}$ et ne se reposent que quelques heures.

En Haïti, l'enfant en domesticité est souvent représenté par un enfant mal coiffé, parfois en haillons, le visage émacié et ayant un seau d'eau sur la tête. Dans certaines familles, le restavè amène l'eau pour tout le monde, mais il n'a pas le droit de l'utiliser, pas même pour sa toilette. Ils ne font pas que des tâches ménagères. En fait, ils participent à tous les travaux qui se font dans la maison : ils participent aux activités commerciales $^{19}$ et agricoles quand cela existe dans la famille de placement; ils font les petites commissions de tous les gens de la demeure; ils gardent les enfants; en ville, ils s'occupent aussi des animaux domestiques de la famille (chats et chiens principalement). C'est, en grande partie, la présence de ces enfants qui permet aux maîtres et maîtresses de maison d'aller travailler ailleurs ou de mener d'autres activités en dehors du toit familial. Tous les membres de la maison progressent, d'une façon ou d'une autre, sauf les domestiques qui doivent espérer qu'un jour on leur tende la main. Ils travaillent beaucoup mais ils n'ont pas le droit de se plaindre. À toutes les activités que mènent ces enfants, il faut ajouter les injures, la bastonnade, l'humiliation, l'ingratitude des maîtres et maîtresses qui ne les remercient presque jamais et qui croient en plus que ce sont eux les éternels ingrats. Les propos de Maurice Sixto dans Ti Sentaniz illustrent bien les conditions de vie des enfants en service en Haïti.

D'autres auteurs ${ }^{20}$ ont décrit les conditions inhumaines dans lesquelles vivent ces enfants en Haïti. D'après J. Despeignes, « ils grandissent la plupart du temps dans l'abjec- tion des humiliations et d'une servitude voisine de l'esclavage... Le maître nourrit son valet pour abuser de sa force de travail autant que dans les formes les plus poussées de l'esclavage... Le domestique est toujours en guenilles, malfamé et traité en objet. Il subit des châtiments corporels pour tout défaut dans l'accomplissement de sa tâche et n'a droit à aucune rétribution. La domesticité est le grand moule où sont coulés les hommes à qui la société n'entend rien donner ».

\section{Les interventions auprès des enfants en domesticité}

Malgré l'ampleur de la domesticité en Haïti, il n'y a presque pas d'interventions en faveur de ces enfants. Maurice Sixto, cité plus haut, grand parolier haïtien, a présenté un sketch intitulé Ti Sentaniz décrivant la situation des enfants en domesticité. À la mort de ce grand parolier, sur l'initiative du Père Jean-Baptiste Miguel, fut créé le foyer Maurice S0ixto. Aujourd'hui, deux foyers sont ouverts : l'un (en 1989) à Carrefour et l'autre à Léogane (en 1994), les deux se trouvent dans le département de l'ouest, au sud de Port-au-Prince. Ces foyers visent :

- l'amélioration du sort des enfants en domesticité en leur offrant une porte sur l'avenir par l'apprentissage d'un métier;

- la mise en confiance de l'enfant et la garantie de sa sécurité affective en servant de pont entre les familles patrons et les familles naturelles;

- la mise d'un frein à la délinquance juvénile en leur offrant un centre d'intérêt propice à leur épanouissement;

- la défense de leurs droits et la garantie de leur intégrité.

À part les foyers Maurice Sixto, les instances de l'État devraient aussi intervenir par le biais soit du service de l'inspection générale du travail du ministère des Affaires sociales (M.A.S) qui doit, d'après l'article 28 du décret créant ce ministère, " contrôler les conditions de travail des femmes et des enfants ", soit du service de la femme et de l'enfant (article 32 du même décret), soit du service de la protection des mineurs de l'Institut du bien-être social de recherche (IBESR) dépendant lui-même du M.A.S (articles 138 à 142 du même décret). Mais dans la réalité, l'IBESR n'est pas fonctionnel et le M.A.S n'intervient pas non plus dans ce problème.

Il est important de noter que la législation sur les enfants en service date de 1961; l'IBESR a été déjà créé, soit en 1958, et le M.A.S en août 1967. En 1983, l'IBESR fut réorganisé par un décret-loi. Celui-ci propose une réor- 
ganisation de l'institution qui devient un organisme technique et administratif du M.A.S chargé entre autres :

- d'accorder une protection particulière à l'enfant, à la femme et à la famille;

- de créer, autoriser, encourager et superviser les œuvres de prévoyance et d'assistance sociale tant publiques que privées.

Des structures étaient déjà en place pour intervenir dans ce problème. Mais, l'IBESR n'a jamais fait grand-chose dans ce sens. Aujourd'hui, plus de 40 ans après la création de cette institution, la situation des enfants en domesticité ne s'est pas améliorée. Vu que le bien-être social à l'enfance est considéré sur le plan de la défense sociale, l'institution s'est mise dans la surveillance et l'application des valeurs « admises » par les groupes dominants. La domesticité n'était pas vraiment une priorité. Il suffit d'encourager que des enfants soient placés quelque part. L'IBESR jouera son rôle de défense et de contrôle sociaux. Il interviendra dans des questions qui n'étaient pas de son ressort. Ce qui lui coûtera sa fermeture. M. Despeignes ${ }^{21}$ écrira que cette fermeture survient parce que l'institution « s'était transformée en une véritable juridiction connaissant même des loyers non payés, avec ses salles de torture et ses hommes de main. Le mariage forcé, destiné a priori à protéger les intérêts moraux des jeunes citadins, devient une entreprise florissante et corruptrice».

Il y a deux ans à peine, on a installé un téléphone d'urgence pour le signalement des mauvais traitements faits aux enfants mis en service particulièrement. Mais on ignore pour le moment les résultats de cette initiative. Compte tenu de la place de l'enfant en domesticité dans l'échelle familiale, il est certain qu'il n'a pas toujours accès au téléphone. Comment avisera-til du mauvais traitement dont il est constamment victime? Est-ce que d'autres personnes le feront? La domesticité est un problème assez délicat dans ce pays où beaucoup de familles disposent d'un ou de plusieurs enfants á leur service. Ces familles n'acceptent pas toujours d'avouer qu'elles ont des enfants en domesticité. Elles prétendent qu'il s'agit d'un petit filleul ou d'une petite filleule, d'un neveu ou d'une nièce ou d'un «petit parent». Et c'est sous le couvert des liens familiaux que se font les mauvais traitements.

\section{Les enfants en domesticité face aux droits de l'enfant} Il est important de noter que Haïti n'a jamais connu d'État providence. Si des enfants sont placés en domesticité, c'est principalement à cause de cette situation. Car la grande majorité des enfants sont mis en service à cause de la situation de misère dans laquelle vivent leurs parents biologiques qui ne bénéficient pas d'aucune allocation et d'aucune aide de la part des responsables. On pourrait tenter de parler de désengagement de l'État face à une telle situation, mais l'État ne s'était jamais engagé directement, malgré la signature ou la ratifica- tion de conventions internationales relatives aux droits et à la protection de l'enfance. Par ailleurs, même quand il existe certaines dispositions légales visant la protection de l'enfance, celles-ci n'ont pas d'effet parce qu'elles ne sont pas respectées. On n'investit pas dans la protection de l'enfance, les enfants en domesticité sont mis dans l'oubli avec des lois qui ne sont pas appliquées. Le signalement pour mauvais traitements infligés aux enfants en Haïti n'est pas connu dans la réalité de ce pays. Même si ces mauvais traitements font la une des médias, les responsables ne font pas grand-chose pour faire respecter les décisions en vigueur et améliorer ainsi le sort des enfants vivant en domesticité. De plus, l'autorité parentale sur les enfants est considérée comme fondamentale dans cette société où « les chefs de famille ont l'obligation de traiter les enfants en domesticité en bons pères de famille ». Suivant le décret-loi du 8 octobre 1982 (article 15 ), « les pères et mères ou la personne qui a la garde de l'enfant peuvent le confier à un centre de rééducation ou, si les motifs de mécontentement sont suffisamment graves, à un centre de détention pour une durée qui ne peut excéder six mois et qui doit être fixée par le doyen du Tribunal civil et le ministère public ». La plupart des parents en Haïti abusent parfois de façon criante de leur autorité et, dans le cas des enfants en domesticité, la situation est pire. Dans des cas d'abus de l'autorité parentale, qui jugera de la gravité des motifs de mécontentement des parents? La loi est muette face à cette question. N'est-ce pas là donner trop de pouvoirs aux parents contre «l'intérêt de l'enfant »?

En ce qui concerne les relations familiales, la plupart des enfants en domesticité dépendent du bon vouloir de leurs patrons et patronnes qui peuvent ou non décider de les envoyer visiter des proches à certaines périodes. Dans la plupart des cas, la famille d'accueil ignore tout des parents de l'enfant qui vit en domesticité chez elle car, bien souvent, les enfants sont passés d'une famille à l'autre, parfois sans leur consentement, comme s'ils étaient des objets. On peut rencontrer des gens qui, à la troisième génération, vivent encore en domesticité. De manière générale, les enfants n'aiment pas ces conditions. Il y en a même qui pleurent à l'idée de vivre dans la domesticité. Contrairement à ce qui est dit dans le droit de l'enfant ${ }^{22}$ sur la défense d'être séparé de ses parents contre sa volonté, dans le cas des enfants en domesticité, c'est monnaie courante. Mais les parents biologiques croient toujours le faire dans l'intérêt de l'enfant face à des familles de placement qui promettent toujours de bien prendre soin de leurs enfants. Les autorités compétentes n'en font rien face à la désolation et à 
la grande tristesse qui envahissent bien souvent les enfants en domesticité.

Pour ce qui est du droit d'expression, de religion et de la liberté de pensée, que peut-on espérer d'un enfant qui est considéré au plus bas de l'échelle familiale et de qui on exige la soumission et l'obéissance? L'enfant en domesticité adopte de fait la religion de ses patrons, sinon il risque de ne jamais avoir du temps pour " pratiquer sa religion ». Même en adoptant celle de ses patrons, il n'est pas sûr de pouvoir la pratiquer, faute de temps.

En ce qui concerne l'éducation, c'est surtout la recherche de la scolarisation qui motive bon nombre de parents à placer leurs enfants en ville ou dans les bourgs. Il existe une forte croyance dans l'institution scolaire qui peut permettre, d'après les parents en Haïti, de sortir de la misère. Schlemmer ${ }^{23}$ rapporte les propos de Fukui pour qui « l'école représente une valeur aux yeux des classes populaires, sans que l'institution scolaire les aide à réaliser celle-ci, au contraire, puisqu'elle entraîne systématiquement l'exclusion de ceux qui ne répondent pas au modèle d'élève qu'elle a elle-même établi ».

En Haïti, la gratuité de l'enseignement est établie depuis 1816 avec la constitution de la même année ${ }^{24}$. Mais ironiquement, la grande majorité des écoles primaires est privée, les écoles secondaires publiques sont assez rares $^{25}$. Il existe diverses petites écoles, dites écoles du soir, et qui sont réservées aux enfants en domesticité. Ce sont les pires écoles de tout le pays. Très peu de ces enfants ont accès à une bonne école, la grande majorité n'y a pas accès. Pour ceux qui y ont accès, le peu de temps consacré aux études est un facteur important dans le redoublement d'une année et le décrochage scolaire. Dans la plupart des familles, le ou la domestique avait commencé l'école avant la naissance des enfants du couple. Mais ces enfants termineront leurs études primaires et même secondaires avant que le domestique atteigne un niveau de scolarisation; il est souvent traité de paresseux, de crétin, de bon à rien ou de quelqu'un qui déteste l'école. En ce sens, les interventions des centres comme les foyers Maurice Sixto, bien qu'elles ne soient que des palliatifs, ont quand même leur place.

Contrairement aux enfants des patrons qui peuvent passer la journée devant la télévision ou à s'amuser autrement, les enfants en domesticité suivent très peu de programmes. Ils sont battus ou injuriés quand ils sont surpris en train de jouer. Dans certaines familles, ils n'ont pas accès à la télé. Pour échapper à cette pression, la plupart d'entre eux perdent beaucoup de temps quand ils sont envoyés faire des commissions en dehors de la maison.

L'exploitation sexuelle des enfants en domesticité est très courante. Certaines domestiques sont souvent violées par leurs patrons qui les abandonnent souvent avec un enfant. Et là encore, rien n'est signalé. Généralement, la patronne s'en prend à la domestique qui, à son avis, a attiré le patron dans son lit par son mauvais comportement.

En Haït, l'exploitation sexuelle des restavèk est l'affaire des hommes. Mais les femmes qui gèrent le quotidien sont le plus souvent responsables des autres types de mauvais traitements infligés à ces enfants (violence verbale et physique, travail forcé...)

Un grand nombre de ces enfants ont préféré vivre dans la rue que de rester en service chez des gens. Lors d'une recherche réalisée en $1991^{26}$, un nombre important d'enfants qui vivaient en domesticité avaient préféré se réfugier dans la rue pour échapper aux mauvais traitements. À cette époque, le problème des enfants de la rue commençait réellement à s'imposer comme problème social $^{27}$.

\section{Conclusions et pistes de recherche}

Déplacés de chez leurs parents biologiques et mis en service très tôt, les enfants en domesticité sont obligés de travailler dans des conditions très inhumaines pour obtenir le minimum nécessaire à leur subsistance. Est-ce une situation conforme à la convention relative aux droits de l'enfant? On peut éventuellement admettre que le travail peut aider à l'épanouissement des individus, mais tel n'est pas le cas pour des enfants en domesticité qui sont exploités, humiliés et marqués par les conditions dégradantes de cette vie. On serait tenté de dire que la domesticité soulage le poids des responsabilités des parents. Mais dans les sociétés, les responsables n'ont-ils pas un mot à dire pour soulager au moins les situations les plus catastrophiques? Il est évident que les restavèk viennent des familles les plus pauvres. Mais il faut noter que ces familles sont plus que pauvres, elles vivent dans la misère.

Comment les enfants vivant en domesticité vivront-ils leur vie adulte? Est-ce que leur progéniture connaîtra un mieux-être dans le futur? Comment ces petites femmes se considèrent-elles après avoir vécu en domesticité? Qu'est-ce qui leur permet de continuer à vivre malgré toutes les mauvaises conditions auxquelles elles font face? Comment réagissent-elles face à la violence une fois devenues adultes? On pourrait multiplier les questions sur l'estime de soi, la violence, la résilience chez les enfants en domesticité; on pourrait penser à des études comparatives par sexe, dans divers pays, etc. Autant de questions auxquelles on n'a pas encore de réponse. Il n'y a pas d'études réalisées sur les retombées et les enjeux de la domesticité juvénile en Haïti. Les chercheurs en sciences sociales et humaines ne se sont pas penchés sur ces cas. Comme le mentionne Bernard Schlemmer ${ }^{28}$ dans le cas des mineurs travailleurs, « il n'existe de par le monde 
qu'un nombre restreint de chercheurs qui se consacrent, pleinement et en tant que chercheurs, à un tel sujet. Le thème relève de l'action, de l'idéalisme : il constituerait donc un problème social, pas une problématique de recherche ».

\section{Notes}

1. Assefa Bequele et Joe Boyden, L'Enfant au travail (Fayard, 1990); Bernard Schlemmer, L'enfant exploité : mise au travail, prolétarisation (Karthala-Osrstom, 1996).

2. Martin Monestier, Les enfants esclaves. L'enfer quotidien de 300 millions d'enfants (Éditeur: Cherche midi, 1998); Assefa Bequele et Joe Boyden 1990, édition Fayard); Bernard Schlemmer, L'enfant exploité, oppression, mise au travail, prolétarisation (KarthalaOrstom, 1996).

3. Institut psycho-social de la famille, Restavèk, la domesticité juvénile en Haïti (commanditée et financée par l'UNICEF, 1998, p. 9).

4. Legrand Bijoux, Coup d'eil sur la famille haïtienne (Éditions des Antilles, 1990).

5. ti moun ki ret kay moun est l'appellation créole qui peut se traduire par enfant demeurant chez des gens.

6. Le restavèk est l'expression créole qui pourrait se traduire par « reste avec». Mais dans la réalité du pays, il est considéré comme une injure grave. Les enfants en domesticité sont souvent qualifiés de restavèk par tous ceux qui veulent les humilier. Le restavèk est considéré comme un moins que rien.

7. Le Moniteur, journal officiel de la République d'Haïti, $n^{\circ} 82 \mathrm{du}$ jeudi 24 novembre 1983 et $\mathrm{n}^{\circ} 18$-A du lundi 5 mars 1984.

8. Le mot est mis entre guillemets parce qu'on doit s'interroger sur ce qu'on appelle des bénéfices dans le cas de ces mineurs travailleurs.

9. Assefa Bequele et Joe Boyden. "Les problèmes et les solutions envisagées" dans L'enfant au travail (étude du BIT 1990, édition Fayard, chapitre 1).

10. Institut psycho-social de la famille. Restavèk, la domesticité juvénile en Haïti (commanditée et financée par l'UNICEF, 1998).

11. Legrand Bijoux, 1990.

12. Montalvo J. Despeignes, Le droit informel haïtien (PUF 1976).

13. Serge Henry-Vieux, "Enfants au travail et cadre juridique de protection" dans Droits de l'homme et Aide à l'enfance Canada: Forum sur enfance et violence (Port-au-Prince, Haïti, 18 et 19 octobre 1995).

14. Claude Meillassoux, "Économie et travail des enfants" dans L'enfant exploité: oppression, mise au travail, prolétarisation (KarthalaOsrstom, 1996).

15. IPSOFA, p. 3.

16. Monestier, p. 35.

17. Droits de l'homme et Aide à l'enfance Canada: Forum sur enfance et violence (Port-au-Prince, Haïti, 18 et 19 octobre 1995).

18. Monestier, 79.

19. Yolande Plumer, Semailles (Haïti, Imprimerie des Antilles, 1970).

20. Montalvo Despeignes, 96; Monestier, 79.

21. Despeignes, 1976, p. 61.
22. Lucie Lamarche et Pierre Bosset, Des enfants et des droits (Presses de l'Université Laval, 1997).

23. Schlemmer, 22.

24. Mathurin Augustin, Assistance publique et privée en Haïti. (Port-au-Prince, Haïti, 1944).

25. Irdèle Lubin, Hacia Un Programa de Educacion Con y Para Los Ninos de la calle de Haïti. (ILCE, México, 1998, thèse de maîtrise en Technologie éducative).

26. Irdèle Lubin, Pour une intégration institutionnelle des enfants de la rue à Port-au-Prince (Port-au-Prince, 1992, mémoire de licence en service social).

27. Lubin, 1992.

28. Schlemmer, 10.

Irdèle Lubin enseigne à la Faculté des Sciences humaines de l'Université d'État d'Haïti. À l'heure actuelle, elle suit un programme de doctorat en Service social à l'Université Laval. Son domaine de recherche est le bien-être à l'enfance. Sa recherche portera sur les enfants de la rue en Haïti. 